\title{
Beyond cultural security; towards sanctuary
}

\author{
Building an oasis in the desert for the health and wellbeing of our children
}

Helen M Milroy MB BS, FRANZCP, CATCAP, Winthrop Professor (Child Psychiatry), ' and

Commissioner, Royal Commission into Institutional Responses to Child Sexual Abuse

1 Faculty of Medicine, Dentistry and Health Sciences, University of Western Australia Perth, WA

helen.milroy@ uwa.edu.au

doi: 10.5694/mjal3.10796

Research p 42

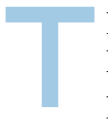
he current state of Aboriginal and Torres Strait Islander health compared with the wider Australian population is well known, with most common health conditions overrepresented, a significant gap in life expectancy, and poorer physical and mental health outcomes. Aboriginal and Torres Strait Islander peoples continue to experience lower levels of access to health services, are more likely to be hospitalised for health conditions, suffer a greater burden of emotional distress than the rest of the population, and are overrepresented in regard to health risk factors such as smoking. ${ }^{1}$ With fewer elders and adults available to buffer families, children and young people often bear the burden of care for sick relatives and are more likely to experience the death of several family members during their developmental stages. Many families will experience multiple life stress events within a relatively short period of time, and the effects of this may be cumulative over generations. $^{2}$ In a study in this issue of the Journal, Askew and colleagues found that urban Aboriginal and Torres Strait Islander children who had experienced significant life stress events had poorer physical health and more parental concern regarding their behaviour. Of note, $51 \%$ of the study participants reported experiencing at least one stressful event. ${ }^{3}$

Recently, the link between stress, development and poor health has been the focus of attention, with an emphasis on promoting good social and emotional wellbeing to enhance development and improve health outcomes. Within the health service environment, culturally appropriate, accessible and secure models of care have been developed to overcome health disparities. But is this extensive knowledge and increasingly sophisticated health system enough to reduce the burden of disease, disadvantage and distress? How can we bring all of this knowledge together to benefit the growth and development of children, enhance their wellbeing and reduce the propensity towards chronic disease and early death?

In the mental health field, the concept of traumainformed care has gained momentum in assisting clinicians to better understand how trauma affects behaviour, recovery and responsiveness within clinical services. As noted by the Mental Health Coordinating Council, trauma-informed care attempts to create "an environment that is more supportive, comprehensively integrated, empowering and therapeu$\mathrm{tic}^{\prime \prime}$. This concept is even more important in regard to children, as we understand the profound impact that trauma can have on the developing brain, memory and self-regulation, as well as attachment relationships and physical health. So how can the health service environment maximise the opportunities to promote resilience, buffer the many traumas Aboriginal and Torres Strait Islander families will face, reduce the secondary impact of trauma in health services, and continue to improve health and wellbeing outcomes?
In 2011, as part of a Yachad Scholarship study tour in Israel, I visited several children's trauma treatment programs and was impressed by the values and attitudes many of the programs had in common. These included believing each child had the capacity for positive change and recovery; the staff accepting both personal and professional responsibility for making the program work for the benefit of the child; having a collective responsibility for all of the nation's children as "family"; having the resourcefulness and flexibility to make things happen if they would benefit the child, such as arranging for music lessons; and never giving up on a child. The belief was often expressed that after what some of these children had been through, they deserved the very best the service could offer. One of the residential services was set up as an oasis in the desert, a place of beauty and tranquillity, yet vibrant and full of life. It was a safe place to be, warm and comforting, but still able to lift you up to see the stars. Every component of the building, landscape and program design was aimed at promoting wellbeing, reducing secondary trauma, empowering recovery and restoring potential. Each child was given the opportunity to choose aspects of his or her treatment, and unique talents and life skills were identified, nurtured and strengthened.

Aboriginal and Torres Strait Islander families will continue to experience stressful life events and adverse health outcomes far in excess of the rest of the population for many years to come. Many children will spend a lot of time in health services, either as clients or with their families. The way children are supported and treated within health services can have a significant influence on their life outcomes, especially given the high burden of risk that is pervasive across the population. Are we, as those charged with providing for their health care needs, able to give them the very best we have to offer during their time with us, through both our professional relationships and the health service environments we provide? Can we continue to build a culturally secure, trauma-informed model of care and provide an oasis in the desert?

Competing interests: No relevant disclosures.

Provenance: Commissioned; not externally peer reviewed.

1 Australian Institute of Health and Welfare. Australia's health 2012. Canberra: AlHW, 2012. (AlHW Cat. No. AUS 156; Australia's Health Series No. 13.)

2 Zubrick SR, Silburn SR, Lawrence DM, et al. The Western Australian Aboriginal Child Health Survey: the social and emotional wellbeing of Aboriginal children and young people. Perth: Curtin University of Technology and Telethon Institute for Child Health Research, 2005. http://aboriginal.childhealth research.org.au/kulunga-research-network/waachs/waachs-volume-2.aspx (accessed Jun 2013).

3 Askew DA, Schluter PJ, Spurling GKP, et al. Urban Aboriginal and Torres Strait Islander children's exposure to stressful events: a cross-sectional study. Med J Aust 2013; 199: 42-45.

4 Bateman J. Trauma informed care and practice. Consultation on the development of a national approach to trauma-informed care \& practice (TICP). Sydney: Mental Health Coordinating Council, 2010. http://www. mhcc.org.au/documents/TICP/TICP-Lit-Review.pdf (accessed Jun 2013). 口 